\title{
Synchronous Type 1 Papillary Renal Cell Carcinoma in a Patient with Rectal Adenocarcinoma
}

\author{
JINHUA PIAO $^{1}$, PAUL FRIEDMAN ${ }^{1}$, SAMEER SIDDIQUI $^{2}$, JULA VEERAPONG $^{2}$ and JIN-PING LAI ${ }^{1}$ \\ Departments of ${ }^{1}$ Pathology, and ${ }^{2}$ Surgery, Saint Louis University School of Medicine, Saint Louis, MO, U.S.A.
}

\begin{abstract}
Synchronous colorectal cancer $(C R C)$ and renal cell carcinoma $(R C C)$ is relatively rare, particularly when the synchronous RCC is of papillary subtype, which is exceedingly rare. We report a case of a 63-year-old Caucasian man with synchronous CRC and type 1 papillary RCC. After the patient presented with three episodes of melena, colonoscopy followed by biopsy confirmed rectal adenocarcinoma. The computed tomographic imaging also showed an incidental mass of the upper pole of the left kidney suspicious for RCC. Once chemoradiation therapy was successfully completed, an ultra low anterior resection and partial nephrectomy were performed concurrently. Histological examination showed colorectal adenocarcinoma (ypT1 NO Mx) and papillary RCC type I (pTla Nx Mx). Although the exact pathogenesis of synchronous CRC and RCC is unknown, it has been suggested that almost all patients with this entity do not have Lynch syndrome. The majority of these patients usually present with CRC-related symptoms and then, during workup, are subsequently found to have an incidental renal mass that is most often diagnosed as clear cell subtype of RCC. To the best of our knowledge, this is only the second reported case of synchronous CRC and type 1 papillary $R C C$.
\end{abstract}

Synchronous primary colorectal carcinoma (CRC) and renal cell carcinoma (RCC) is relatively rare $(0.4-4.85 \%)(1-5)$. Most studies believe the true incidence to be closer to $0.4-$ $0.5 \%$, with only one study of five cases reporting a $4.85 \%$ incidence rate (1). Synchronous cancers are defined as two or more primary cancers diagnosed in the same patient at the same time or within 6 months of each other $(6,7)$. Although

Correspondence to: Jin-Ping Lai, MD, Ph.D., Department of Pathology, Saint Louis University School of Medicine, 1402 S. Grand Blvd., St Louis, MO 63104, U.S.A. Tel: +1 3145778475, Fax: +1 3145776132, e-mail: jinpinglai@slu.edu

Key Words: Type 1 papillary renal cell carcinoma, synchronous tumor, rectal adenocarcinoma. the exact pathogenesis of this entity remains to be elucidated, immunohistochemical studies suggest that synchronous CRC and RCC develop independently of microsatellite instability, and that patients with this entity have microsatellite-stable tumors and do not have Lynch syndrome $(10,11)$. Studies suggest that patients with this entity have a higher risk of developing other malignancies (11), and the possibility of a shared genetic cause warrants further studies.

In this report, we describe the case of a 63-year-old Caucasian man with synchronous rectal adenocarcinoma and type 1 papillary RCC. The literature is reviewed and the pathogenesis and prognosis of this entity are discussed.

\section{Case Report}

A 63-year-old Caucasian man who presented with three episodes of melena was referred for colonoscopy, that revealed multiple colonic polyps as well as an ulcerated rectal mass.

The patient's medical and social history was significant for type II diabetes mellitus and occasional cigar smoking. He also had a significant family history of cancer, with his father having been diagnosed with kidney and bladder cancer, his brother with a rare lymphoma, and his son with a germ-cell tumor.

A biopsy of the rectal mass was performed, and histopathological examination confirmed CRC. On further workup, computed tomographic imaging revealed a $7.2 \times 6.9 \times 5.5 \mathrm{~cm}$ cystic lesion in the upper pole of the left kidney with an associated $2.2 \times 1.9 \times 1.4 \mathrm{~cm}$ solid enhancing component (Figure 1). A positron-emission tomographic scan revealed a fluorodeoxyglucose-avid rectal mass. No distant metastasis or lymphadenopathy was identified.

Our patient successfully completed neoadjuvant chemoradiation therapy for his CRC. He was subsequently admitted for ultra low anterior resection with a diverting loop ileostomy and left partial nephrectomy. Both specimens from the ultra low anterior resection and left partial nephrectomy were obtained by our Department of Pathology for gross and histopathological examination: 
The ultra low anterior resection specimen consisted of a segment of rectosigmoid colon $(32.0 \mathrm{~cm}$ in length $\times 5.0 \mathrm{~cm}$ in diameter). A $1.7 \times 1.2 \mathrm{~cm}$ firm, tan-pink ulcerated area $(2.5$ $\mathrm{cm}$ from the distal surgical margin) and a sessile polypoid lesion $(0.2 \mathrm{~cm}$ in greatest dimension, $9.0 \mathrm{~cm}$ from the distal surgical margin) were identified (Figure 2A). After processing, 12 lymph nodes were found in the pericolonic adipose tissue. Microscopic examination revealed a lowgrade (well- to moderately differentiated) residual rectal adenocarcinoma, with invasion into the submucosa (Figure 2B). No lymphovascular invasion or perineural invasion was identified. Both proximal and distal surgical resection margins were free of tumor. Immunohistochemically, the tumor cells showed strong immunoreactivity for caudalrelated homeobox gene 2 (CDX2) (Figure 2C) and cytokeratin 20 (CK20) (Figure 2D left), and was negative for cytokeratin 7 (CK7) (Figure 2D right) and renal cell carcinoma marker.

The left partial nephrectomy specimen consisted of a $5.2 \times 2.6 \times 0.8 \mathrm{~cm}$, unoriented portion of kidney with a simple cyst overlying a $2.4 \times 1.9 \times 0.8 \mathrm{~cm}$ yellow-tan solid mass. Microscopic examination revealed a type 1 papillary RCC with a Fuhrman nuclear grade of 1-2 out of 4 . The tumor comprised of tightly packed papillae, resulting in a 'solid' appearance. Some of the fibrovascular cores contained clusters of foamy macrophages and were lined by a single layer of tumor cells with hyperchromatic nuclei and inconspicuous nucleoli. Scattered psammoma bodies were present. No lymphovascular invasion or perineural invasion were identified. The tumor was limited to the kidney, and the parenchymal surgical resection margin was uninvolved. Immunohistochemically, the tumor cells showed strong immunoreactivity for CK7, focal and weak immunoreactivity for renal cell carcinoma marker, and negative immunoreactivity for CK20, CDX2, and thyroid transcription factor 1 (Figure 3).

\section{Discussion}

Synchronous malignancies were first described by Billroth in 1889 (8). Then in 1932, Warren and Gates published a detailed study of post-mortem examination on multiple malignancies, and established the most commonly accepted criteria: each tumor must arise from different locations, have distinct histology, and the possibility of one being a metastasis of the other must be excluded (9). Synchronous CRC and RCC in our patient meet the diagnostic criteria for the diagnosis of multiple primary malignant neoplasms, which were established by Warren and Gates (9).

Although the exact pathogenesis is unknown, studies suggest that synchronous CRC and RCC develop independently of a mismatch repair (MMR) defect. Cullinane et al. reported that among seven patients with both CRC and $\mathrm{RCC}$, only five CRC tumor blocks and six RCC tumor blocks

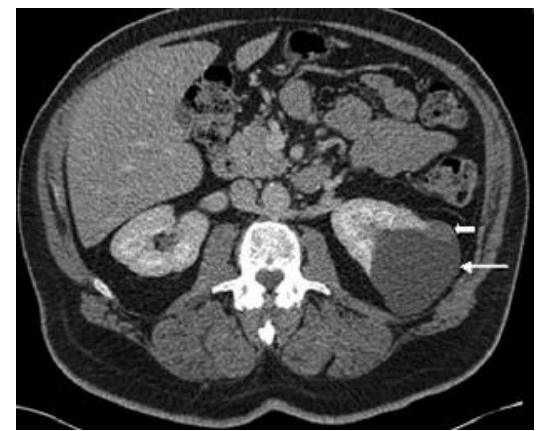

Figure 1. Computed tomographic image of the abdomen showing a $7.2 \times 6.9 \times 5.5 \mathrm{~cm}$ cystic lesion in the superior pole of the left kidney (thin arrow) with an associated $2.2 \times 1.9 \times 1.4 \mathrm{~cm}$ solid enhancing component (broad arrow).

were available for evaluation. Four out of five rectal cancers and five out of the six RCC cancers were microsatellite-stable (10). A cohort study by Steinhagen et al. reported similar findings. Among the tissue blocks available for immunohistochemical stains from 10 patients with both CRC and RCC, nine patients had normal expression of all four MMR proteins in the CRC specimens, and one patient showed an absence of the muts homolog 6 (MSH6) protein, however, his RCC specimen showed normal expression of all four MMR proteins (11). Moreover, clinicopathological data suggested that patients with synchronous CRC and RCC have an increased risk of developing other malignancies, and so the possibility of a genetic etiology has been suggested for this entity. This warrants carrying out larger studies in order to determine whether there is a potential underlying genetic cause. Among the 101 patients who were included in the study by Steinhagen et al., $42 \%$ of the patients had synchronous CRC and RCC. In this cohort study, 32\%, 7\%, and $3 \%$ of the patients had one, two, and three additional primary malignancies respectively (11).

Although clear cell RCC is the most common subtype of $\mathrm{RCC}$, it has been suggested that papillary RCCs are more likely associated with secondary malignancies, such as CRC and prostate cancer, when compared to patients with clear cell RCCs. Among the 2,722 patients Thompson et al. studied, 2,188 (80.4\%), 378 (13.9\%), and $128(4.7 \%)$ of patients had clear cell, papillary, and chromophobe type RCCs, respectively. Of these cases, 34 (1.6\%) of the patients with clear cell RCC, 9 (2.4\%) of those with papillary RCC, and $5(3.9 \%)$ of those with chromophobe RCC had metachronous CRC (12).

Asymptomatic patients with incidentally discovered RCCs have significantly higher survival rates compared to symptomatic patients. Tsui et al. showed that patients with incidental RCCs had a 5-year survival rate of $85.3 \%$, but the rate was only $62.5 \%$ among patients with symptomatic 


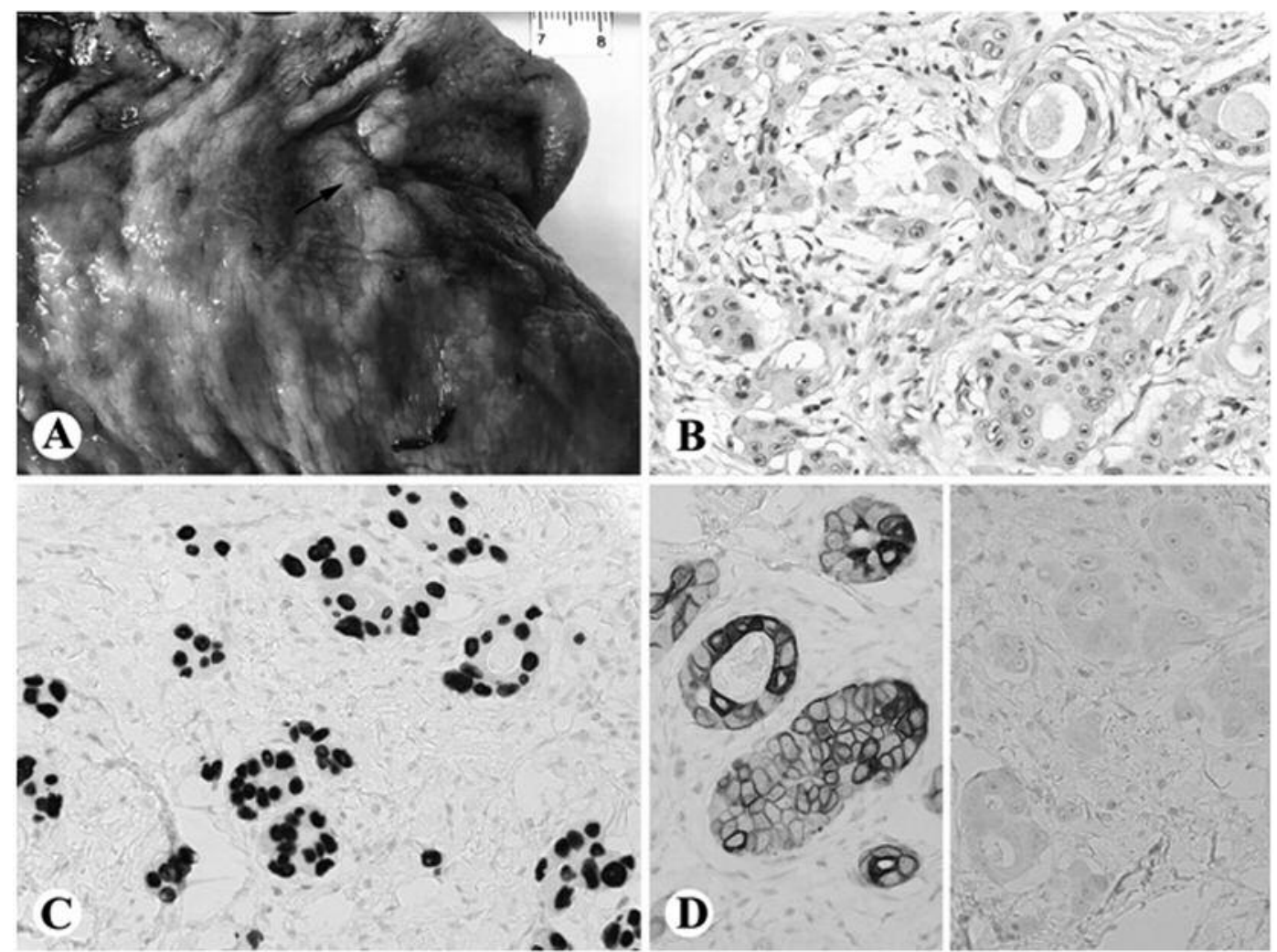

Figure 2. A: Gross examination of the rectosigmoid colon showing a $1.7 \times 1.2 \mathrm{~cm}$ firm, tan-pink ulcerated area; B: Hematoxylin and eosin staining of the specimen showing a low-grade (well-to moderately differentiated) residual rectal adenocarcinoma (x400). C: Tumor cells showed strong immunoreactivity for caudal-related homeobox gene 2 (x400). D: Tumor cells also showed strong immunoreactivity for cytokeratin 20 (left), but no immunoreactivity for cytokeratin 7 (right) $(\times 400)$.

RCCs. The asymptomatic RCCs had significantly lower histologic grade and stage, no recurrence after resection, and only $2.1 \%$ of the patients had distant metastasis (13). Linehan et al. reported similar results (14). On the contrary, symptomatic RCCs had significantly higher histological grade and stage, $0.4 \%$ local tumor recurrence, and $8.2 \%$ of the patients had distant metastasis.

\section{Conclusion}

Our report describes a patient with synchronous CRC and type 1 papillary RCC. It is important to recognize this entity for the following reasons: i) It has been suggested that patients with synchronous CRC and RCC have an increased risk of developing other malignancies. ii) The majority of these RCCs are clear-cell type and incidentally discovered during workup for CRC. Incidentally found RCC has a better prognosis and higher 5-year survival rate because of significantly lower grade and stage compared to symptomatic RCC. iii) Patients with papillary or chromophobe RCC may be more likely to develop metachronous CRC compared to patients with clear cell RCC. Therefore, it is important for clinicians to be aware of the possibility of synchronous malignancies when a CRC or RCC is discovered, particularly if the RCC is of papillary or chromophobe subtype, which has been shown to have a higher likelihood of this process. If the RCC is incidentally discovered and is of low nuclear grade and stage, the outcome and survival rate related to that particular malignancy will be better.

\section{Conflicts of Interest}

The Authors declare that they have no financial conflicts of interest in regard to this report.

\section{References}

1 Halak M, Hazzan D, Kovacs Z and Shiloni E: Synchronous colorectal and renal carcinomas: a noteworthy clinical entity. Report of five cases. Dis Colon Rectum 43: 1314-1315, 2000.

2 Capra F, Scintu F, Zorcolo L, Marongiu L and Casula G: Synchronous colorectal and renal carcinomas. Is it a definite clinical entity? Chir Ital 55: 903-906, 2003. 

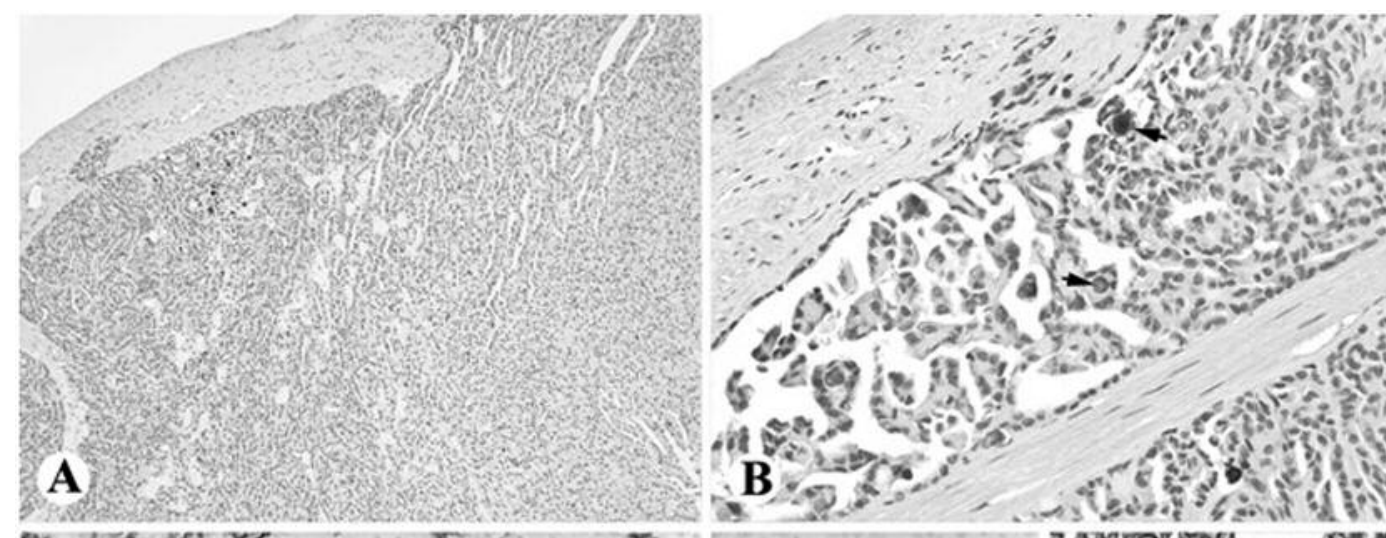

A
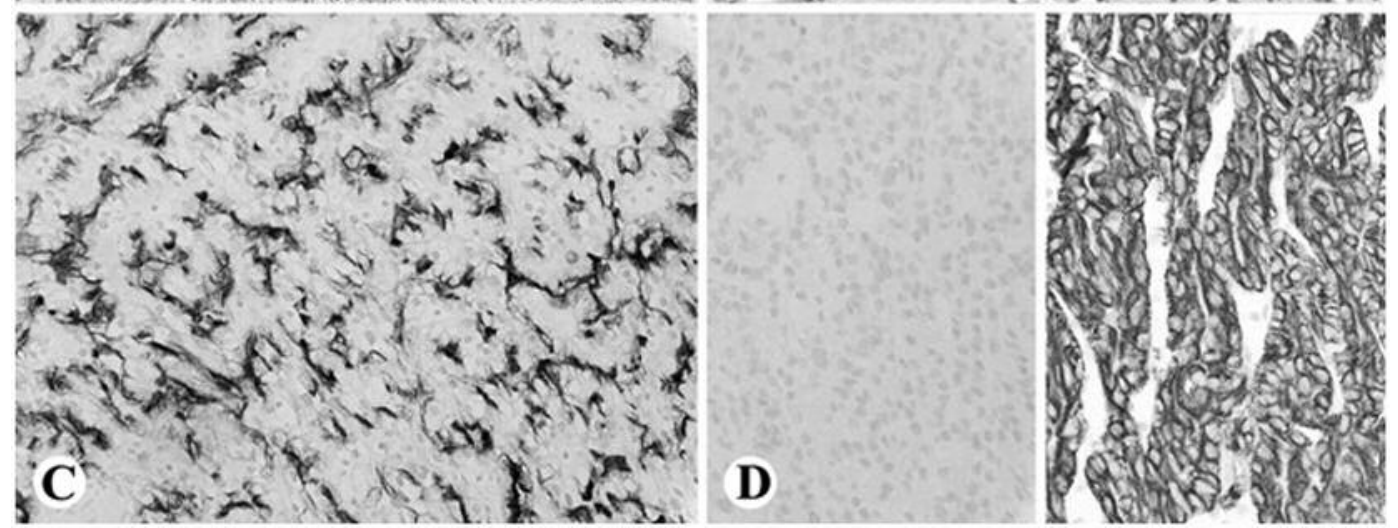

Figure 3. A, B: Hematoxylin and eosin staining showing papillary renal cell carcinoma, type 1, Fuhrman nuclear grade 1-2. The tumor cells have formed single layers of cells lining the fibrovascular cores, and the nuclei were hyperchromatic with inconspicuous nucleoli. Psammoma bodies are present $(A: \times 100 ; B: \times 400)$. C: The tumor cells were positively immunoreactive for immunohistochemical stain for renal cell carcinoma marker (×400). D: Tumor cells showed no immunoreactivity for cytokerain 20 (left) but strong immunoreactivity for cytokeratin 7 (right) (x400).

3 Polk HC, Sprrat JS and Butcher HR: Frequency of multiple primary malignant neoplasms associated with colorectal carcinoma. Am J Surg 109: 71-75, 1965.

4 Sprrat JS Jr. and Hoag MG: Incidence of multiple primary cancers per man-year of follow-up. 20-year review from the Ellis Fischel State Cancer Hospital. Ann Surg 164: 775-784, 1966.

5 O'Boyle KP and Kemeny N: Synchronous colon and renal cancers: six cases of a clinical entity. Am J Med 87: 691-693, 1989.

6 Grace S, Muzaffar R, Veerapong J, Alkaade S, Poddar N, Guzman M, Batanian J, Vogler C and Lai JP: Synchronous quadruple primary neoplasms: glioblastoma, neuroendocrine tumor, schwannoma and sessile serrated adenoma in a patient with a history of prostate cancer. Anticancer Res 35: 2121-2127, 2015.

7 Meeks MW, Grace S, Chen Y, Petterchak J, Bolesta E, Zhou Y and Lai JP: Synchronous quadruple primary neoplasms: colon adenocarcinoma, collision tumor of neuroendocrine tumor and Schwann cell hamartoma and sessile serrated adenoma of the appendix. Anticancer Res 36: 4307-4311, 2016.

8 Billroth T: Die allgemeine chirurgishe Pathologie and Therapie in 51 Vorlesungen. Berlin, Germany, G. Reimer, pp. 908, 1889.

9 Warren S and Gates O: Multiple primary malignant tumors: a survey of the literature and a statistical study. Am J Cancer 16: $1358-1414,1932$.
10 Cullinane CA, Ellenhorn JD and Weitzel JN: Microsatellite instability is a rare finding in tumors of patients with both primary renal and rectal neoplasms. Cancer Genet Cytogenet 148: 163-165, 2004.

11 Steinhagen E, Moore HG, Lee-Kong SA, Shia J, Eaton A, Markowitz AJ, Russo P and Guillem JG: Patients with colorectal and renal cell carcinoma diagnoses appear to be at risk for additional malignancies. Clin Colorectal Cancer 12: 23-27, 2013.

12 Thompson RH, Leibovich BC, Cheville JC, Webster WS, Lohse $\mathrm{CM}$, Kwon ED, Zincke $\mathrm{H}$ and Blute ML: Second primary malignancies associated with renal cell carcinoma histologic subtypes. J Urol 176: 900-904, 2006.

13 Tsui KH, Shvarts O, Smith RB, Figlin R, de Kernion JB and Belldegrun A: Renal cell carcinoma: prognostic significance of incidentally detected tumors. J Urol 163: 426-430, 2000.

14 Linehan WM, Shipley WU and Parkinson OR: Cancer of the kidney and ureter. In: Cancer: Principles and Practice of Oncology. Fifth Edition. Devita VT Jr., Hellman S and Rosenberg SA (eds.). Philadelphia: Lippincott-Raven, pp. 1271-300, 1997.

Received July 12, 2016

Revised August 2, 2016 Accepted August 9, 2016 